\title{
Multi-sensor State Estimation over Lossy Channels using Coded Measurements
}

\author{
Tianju Sui ${ }^{\text {a }}$, Damian Marelli ${ }^{\text {b,c,* }}$, Ximing Sun ${ }^{\text {a }}$, Minyue Fu ${ }^{\text {d,b }}$ \\ ${ }^{a}$ School of Control Science and Engineering, Dalian University of Technology, Dalian, China. \\ ${ }^{\mathrm{b}}$ School of Automation, Guangdong University of Technology, Guangzhou, China. \\ ${ }^{\mathrm{c}}$ French Argentine International Center for Information and Systems Sciences, National Scientific and Technical Research \\ Council, Argentina. \\ ${ }^{\mathrm{d}}$ School of Electrical Engineering and Computer Science, The University of Newcastle, NSW 2308, Australia.
}

\begin{abstract}
This paper focuses on a networked state estimation problem for a spatially large linear system with a distributed array of sensors, each of which offers partial state measurements, and the transmission is lossy. We propose a measurement coding scheme with two goals. Firstly, it permits adjusting the communication requirements by controlling the dimension of the vector transmitted by each sensor to the central estimator. Secondly, for a given communication requirement, the scheme is optimal, within the family of linear causal coders, in the sense that the weakest channel condition is required to guarantee the stability of the estimator. For this coding scheme, we derive the minimum mean-square error (MMSE) state estimator, and state a necessary and sufficient condition with a trivial gap, for its stability. We also derive a sufficient but easily verifiable stability condition, and quantify the advantage offered by the proposed coding scheme. Finally, simulations results are presented to confirm our claims.
\end{abstract}

Key words: Networked state estimation, Sensor fusion, Packet loss, Minimum mean-square error.

\section{Introduction}

This work is concerned with the sensor fusion problem over lossy channels. Each sensor obtains a partial state measurement subject to some additive noise, and transmits it to a remote (central) estimator through a communication network involving packet loss. The estimator computes a minimum mean-square error (MMSE) estimate of the system state using the received measurements. The configuration is illustrated in Fig. 1. This setup is motivated by a wide range of applications including networked control systems, multi-agent systems, smart electricity networks and sensor networks $[1,2]$.

The problem of networked state estimation, based on MMSE estimation, has received significant attention in

\footnotetext{
* Corresponding author.

Email addresses: suitj@mail.dlut.edu.cn (Tianju Sui), Damian.Marelli@newcastle.edu.au (Damian Marelli), sunxm@dlut.edu.cn (Ximing Sun), minyue.fu@newcastle.edu.au (Minyue Fu).

1 This work was not presented at any conference.
}

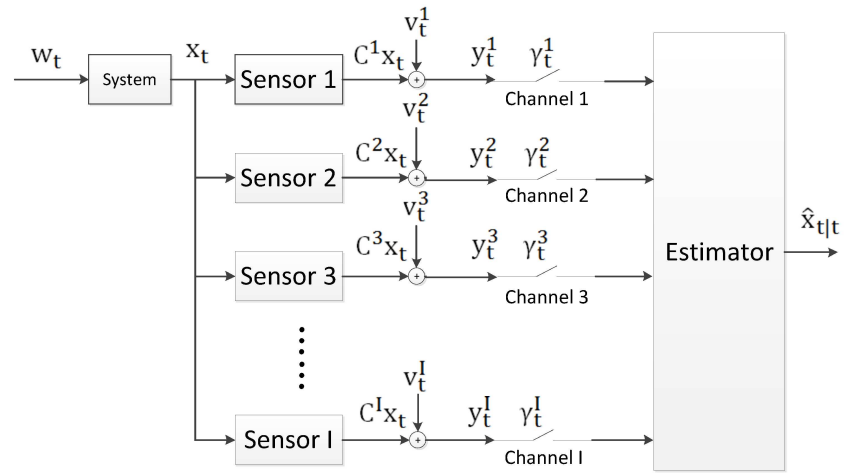

Fig. 1. Networked state estimation using raw measurements.

recent years $[3,4,5,6]$. One of the major difficulties comes from the packet loss occurring while transmitting sensor measurements. A central problem consists in determining the packet loss statistics required to guarantee the stability of the MMSE estimator. This was done in [3] for the case in which the packet loss is independent and identically distributed. This result has been generalized 
to different packet loss models and algebraic system's structure in $[7,8,9,10,11,12,13,14]$. The most general result within this line was recently reported in [15], where the authors state a conceptional necessary and sufficient condition with a trivial gap, for general packet loss statistics and system structure.

The above works assume that raw measurements without preprocessing are transmitted to the estimator. It turns out that the use of preprocessing can relax the channel requirements, in terms of channel statistics, needed to guarantee stability $[16,17]$. For example, in [18], the sensor locally obtains a MMSE estimate and transmits it instead of its measurement. A drawback of this approach is that this increases the amount of communications, because the estimated state needs to be transmitted, which typically has a higher dimension than the raw measurement. To rectify this, a coded measurement $[19,20]$ is built by using a linear combination of the most recent measurements within a coding window, and this is transmitted instead of the raw measurement $[21,22]$.

The works described so far consider the case in which a single sensor transmits over a single channel. In many applications, the system whose state needs to be estimated covers a wide geographical area. Such a largescale system is typically equipped with multiple sensors for measurements. The state estimation problem resulting from this setup has been studied in a number of works $[23,24,25,26,27,28,29]$. In a sensor network setup, all the sensors can transmit their measurements to a central estimator over different channels, each with its own packet loss statistics. Conditions for guaranteeing stability in this network setup can be very complex, and may be very strong for certain systems, as reported in $[26,27,28,30]$.

In [15], the authors derived a necessary and sufficient condition, having a trivial gap, for the stability of a MMSE estimator. These condition is stated in very general terms, so it can be applied in a wide range of settings. In the present work, we make use of this result to design a MMSE estimator for a multi-sensor network problem. Our contributions are the following: (1) In the context of this work, the stability of the estimator depends on how reliable are the communication channels between each sensor and the estimator. We propose a coding scheme that, while reducing the amount of transmitted data, i.e., the dimension of the coded vector transmitted by each sensor at each time step, achieves the weakest requirement on the channel reliability required to guarantee stability. (2) While the aforementioned condition is necessary and sufficient, its computation can be mathematically involved is some cases. To go around this, we also provide a sufficient condition for easier computation. (3) We quantify the gain, in terms of channel reliability, offered by the proposed coding scheme, when compared with the scheme using raw measurements.
The rest of the paper is organized as follows. In Section 2 we describe the system, channel and coding models. In Section 3, we derive the expression of the state estimator using coded measurements. In Section 4.1 we provide a necessary and sufficient condition with a trivial gap for the stability of the MMSE estimator. In Section 4.2 we derive a simpler sufficient condition for its stability. In Section 5 we derive a necessary and sufficient condition with a trivial gap for the stability of the MMSE estimator using raw measurements, and quantify the advantage offered by the proposed coding scheme. We give simulation results illustrating our claims in Section 6, and give concluding remarks in Section 7. To improve readability, some proofs are given in the Appendix.

Notation 1 The sets of real and natural numbers are denoted by $\mathbb{R}$ and $\mathbb{N}$, respectively. We use $\mathbb{P}(\mathcal{S})$ to denote the probability of the set $\mathcal{S}$ and $\mathbb{E}(x)$ to denote the expected value of the random variable $x$. For a vector or matrix $x$ we use $x^{\top}$ to denote its transpose. We use $I_{d}$ to denote the $d$-dimensional identity matrix and I to denote the same matrix when the dimension is clear from the context.

\section{Problem statement}

Consider a discrete-time stochastic system

$$
x_{t+1}=A x_{t}+w_{t},
$$

where $x_{t} \in \mathbb{R}^{n}$ is the system state and $w_{t} \sim \mathcal{N}(0, Q)$ is white Gaussian noise with $Q \geq 0$. The initial time is $t_{0}$ and the initial state is $x_{t_{0}} \sim \overline{\mathcal{N}}\left(\bar{x}_{t_{0}}, P_{t_{0}}\right)$, with $P_{t_{0}} \geq 0$. A sensor network with $I$ nodes, as depicted in Fig. 1, is used to measure the state in a distributed manner. For each $i \in\{1, \cdots, I\}$, the measurement $y_{t}^{i} \in \mathbb{R}^{m_{i}}$ obtained at sensor $i$ is given by

$$
y_{t}^{i}=C^{i} x_{t}+v_{t}^{i}
$$

where $v_{t}^{i} \sim \mathcal{N}\left(0, R^{i}\right)$ is $m_{i}$-dimensional white Gaussian noise with $R^{i}>0$. Let $m=\sum_{i=1}^{I} m_{i}$ and $C^{\top}=$ $\left[C^{1 \top}, \cdots, C^{I \top}\right] \in \mathbb{R}^{n \times m}$. We assume that $(A, C)$ is detectable and $x_{0}, w_{t}, v_{t}^{i}$ are jointly independent.

We are concerned with a networked estimation system, where each sensor is linked to the central estimator through a communication network. Due to the channel unreliability, the transmitted packets may be randomly lost. We use a binary random process $\gamma_{t}^{i}$ to describe the packet loss process. That is, $\gamma_{t}^{i}=1$ indicates that the packet from sensor $i$ is successfully delivered to the estimator at time $t$, and $\gamma_{t}^{i}=0$ indicates that the packet is lost. We assume that the random variables $\gamma_{t}^{i}$, $t \in \mathbb{N}, i \in\{1, \cdots, I\}$ are independent and identically distributed (i.i.d). Also, for each $i \in\{1, \ldots, I\}, p_{i}=\mathbb{E}\left[\gamma_{t}^{i}\right]$.

As a consequence of packet loss, the estimator may fail to generate a stable state estimate. To improve the stability, 
instead of transmitting the raw measurements from each sensor, we encode them before transmission, as depicted in Fig 2. More precisely, for a given coding window length $L \in \mathbb{N}$, the coded measurement of sensor $i$ at time $t$, after going through the channel, is given by

$$
z_{t}^{i}=\sum_{l=1}^{L} \gamma_{t}^{i} H_{t, l}^{i \top} y_{t-l+1}^{i} \in \mathbb{R}^{c_{i}},
$$

for some coding weight matrices $H_{t, l}^{i} \in \mathbb{R}^{c_{i} \times m_{i}}, l \in$ $\{1, \cdots, L\}$, with $c_{i} \leq m_{i}$, and the convention that $y_{t}=0$ for $t \leq t_{0}$.

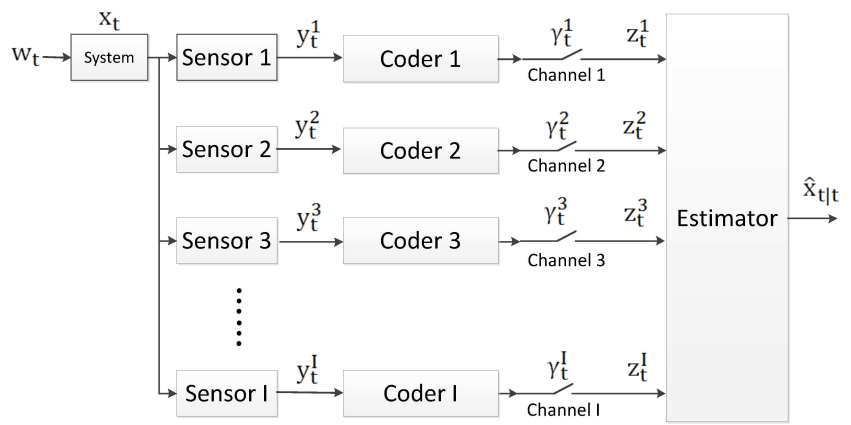

Fig. 2. Networked state estimation using coded sensor measurements.

Remark 2 The coding scheme described in (3) allows reducing the dimension of the transmitted information from $m_{i}$ to $c_{i}$, to the extent to which even a scalar $\left(c_{i}=1\right)$ can be transmitted. This obviously reduce the communication load. We will show in Section 5 that the coding scheme can improve the stability of the state estimator with any choice of $1 \leq c_{i} \leq m_{i}$.

To represent the packet loss process for all sensors at time $t$, we introduce

$$
\begin{aligned}
& \Gamma_{t}=\operatorname{diag}\left\{\Gamma_{t}^{1}, \cdots, \Gamma_{t}^{I}\right\} \in \mathbb{D}, \\
& \Gamma_{t}^{i}=\gamma_{t}^{i} I_{c_{i}},
\end{aligned}
$$

where $\mathbb{D}$ consists of the $2^{I}$ matrices resulting from all possible values of $\gamma_{t}^{i}$. The information available to the estimator from time $t_{0}$ to $t$ is then given by

$$
\mathcal{F}_{t_{0}, t}=\left\{\left(\Gamma_{t_{0}}, \Gamma_{t_{0}} z_{t_{0}}\right), \cdots,\left(\Gamma_{t}, \Gamma_{t} z_{t}\right)\right\}
$$

where $z_{t}^{\top}=\left[z_{t}^{1 \top}, \cdots, z_{t}^{I \top}\right]$. Using this information, the MMSE estimator computes

$$
\hat{x}_{t \mid t-1}\left(\mathcal{F}_{t_{0}, t}\right)=\mathbb{E}\left[x_{t} \mid \mathcal{F}_{t_{0}, t}\right]
$$

Its prediction error covariance is defined by

$$
P_{t \mid t-1}\left(\mathcal{F}_{t_{0}, t}\right)=\mathbb{E}\left[\left(x_{t}-\hat{x}_{t \mid t-1}\right)\left(x_{t}-\hat{x}_{t \mid t-1}\right)^{\top} \mid \mathcal{F}_{t_{0}, t}\right] .
$$

Definition 3 We say that the estimator is stable if [3]

$$
\sup _{\substack{t_{0} \in \mathbb{Z} \\ P_{t_{0}} \geq 0}} \limsup _{t \rightarrow \infty}\left\|\mathbb{E}\left(P_{t+1 \mid t}\left(\mathcal{F}_{t_{0}, t}\right)\right)\right\|<\infty
$$

As it is known $[3,18,14]$, when the spectral radius of $A$ is greater than one, the packet loss can lead to an unstable estimator. Our goal is to $\operatorname{design} L$ and $H_{t}, t \in \mathbb{N}$, to make the stability condition as weak as possible. In doing so, we also provide expressions for $\hat{x}_{t \mid t-1}$ and $P_{t \mid t-1}$. Notice that, to simplify the notation, we use $\hat{x}_{t \mid t-1}$ and $P_{t \mid t-1}$ in place of $\hat{x}_{t \mid t-1}\left(\mathcal{F}_{t_{0}, t}\right)$ and $P_{t \mid t-1}\left(\mathcal{F}_{t_{0}, t}\right)$. We will use this notation in the rest of the paper.

\section{The MMSE state estimation}

In this section we assume that $L$ and $H_{t, l}^{i}, \forall t, l, i$ are given, and derive the expressions of $\hat{x}_{t \mid t-1}$ and $P_{t \mid t-1}$.

Recall that $C^{\top}=\left[C^{1 \top}, \cdots, C^{I \top}\right] \in \mathbb{R}^{n \times m}$ and define $y_{t}^{\top}=\left[y_{t}^{1 \top}, \cdots, y_{t}^{I \top}\right] \in \mathbb{R}^{1 \times m}$ and $v_{t}^{\top}=$ $\left[v_{t}^{1 \top}, \cdots, v_{t}^{I \top}\right] \in \mathbb{R}^{1 \times m}$. We can then rewrite (2) as

$$
y_{t}=C x_{t}+v_{t}
$$

Let $c=\sum_{i=1}^{I} c_{i}, H_{t, l}=\operatorname{diag}\left(H_{t, l}^{1} \cdots H_{t, l}^{I}\right) \in \mathbb{R}^{c \times m}$ and $H_{t}=\left[H_{t, 1}, \cdots, H_{t, L}\right] \in \mathbb{R}^{c \times m L}$. Let also $\Gamma_{t}^{i}=\gamma_{t}^{i} I_{c_{i}}$ and $\Gamma_{t}=\operatorname{diag}\left\{\Gamma_{t}^{1}, \cdots, \Gamma_{t}^{I}\right\}$, and recall that $z_{t}^{\top}=$ $\left[z_{t}^{1 \top}, \cdots, z_{t}^{I \top}\right] \in \mathbb{R}^{1 \times c}$. We can then rewrite (3) as

$$
z_{t}=\Gamma_{t} H_{t}\left[y_{t}^{\top}, \cdots, y_{t-L+1}^{\top}\right]^{\top}
$$

Let $u_{t}^{\top}=\left[x_{t}^{\top}, y_{t}^{\top}, \cdots, y_{t-L+1}^{\top}\right] \in \mathbb{R}^{1 \times(n+m L)}$. We can obtain a state-space representation of (3) as follows

$$
\begin{aligned}
u_{t+1} & =\bar{A} u_{t}+\epsilon_{t}, \\
z_{t} & =\bar{D}_{t} u_{t},
\end{aligned}
$$

where

$$
\begin{aligned}
\bar{A} & =\left[\begin{array}{ccc}
A & 0 & 0 \\
C A & 0 & 0 \\
0 & I & 0
\end{array}\right], \quad \epsilon_{t}=\left[\begin{array}{c}
w_{t} \\
C w_{t}+v_{t+1} \\
0
\end{array}\right], \\
\bar{D}_{t} & =\Gamma_{t}\left[0 H_{t}\right]=\left[\bar{D}_{t}^{1}, \cdots, \bar{D}_{t}^{I}\right] .
\end{aligned}
$$

Thus, the expressions of $\hat{x}_{t \mid t-1}$ and $P_{t \mid t-1}$ can be derived by running a Kalman filter[31,3] on (4)-(5). The resulting 
estimator is given by the following recursions

$$
\begin{aligned}
\hat{x}_{t \mid t-1} & =[I, 0] \hat{u}_{t \mid t-1}, \\
P_{t \mid t-1} & =[I, 0] \Sigma_{t \mid t-1}[I, 0]^{\top},
\end{aligned}
$$

where

$$
\begin{aligned}
\hat{u}_{t+1 \mid t} & =\bar{A} \hat{u}_{t \mid t}, \\
\hat{u}_{t \mid t} & =\hat{u}_{t \mid t-1}+\sum_{i=1}^{I} \gamma_{k}^{i} K_{t}^{i}\left(z_{t}^{i}-\bar{D}_{t}^{i} \hat{u}_{t \mid t-1}\right), \\
\Sigma_{t+1 \mid t} & =\bar{A} \Sigma_{t \mid t} \bar{A}^{\top}+\bar{Q}, \\
\Sigma_{t \mid t} & =\left(I-\sum_{i=1}^{I} \gamma_{k}^{i} K_{t}^{i} \bar{D}_{t}^{i}\right) \Sigma_{t \mid t-1},
\end{aligned}
$$

with

$$
\begin{aligned}
K_{t}^{i} & =\Sigma_{t \mid t-1} \bar{D}^{i \top}\left(\bar{D}^{i} \Sigma_{t \mid t-1} \bar{D}^{i \top}\right)^{\dagger}, \\
\bar{Q} & =\left[\begin{array}{ccr}
Q & Q C^{\top} & 0 \\
C Q & C Q C^{\top}+R & 0 \\
0 & 0 & 0
\end{array}\right],
\end{aligned}
$$

and initialized by

$$
\hat{u}_{t_{0}+1 \mid t_{0}}=0, \quad \Sigma_{t_{0}+1 \mid t_{0}}=\left[\begin{array}{ccc}
P_{t_{0}} & P_{t_{0}} \bar{D}_{t_{0}}^{\top} & 0 \\
\bar{D}_{t_{0}} P_{t_{0}} & \bar{D}_{t_{0}} P_{t_{0}} \bar{D}_{t_{0}}^{\top} & 0 \\
0 & 0 & 0
\end{array}\right] .
$$

\section{Stability Analysis for the MMSE estimator}

In this section we provide conditions for the stability of the MMSE estimator derived in Section 3. In Section 4.1 we provide a necessary and sufficient condition with a trivial gap, and give the values of the design parameters $L$ and $H_{t}, t \in \mathbb{N}$, making this condition as weak as possible. In Section 4.2 we provide a sufficient condition for stability which is easier to verify.

\subsection{Necessary and sufficient condition}

In this section we state a necessary and sufficient condition, having a trivial gap, for the stability of the MMSE estimator using coded measurements. We then design $L$ and $H_{t}, t \in \mathbb{N}$ to make this condition as weak as possible.

Let $\bar{A}=\bar{T} \bar{J} \bar{T}^{-1}$ be the Jordan normal form of $\bar{A}$. We can then write (4)-(5) in Jordan canonical form as

$$
\begin{aligned}
\tilde{u}_{t+1} & =\bar{J} \tilde{u}_{t}+\tilde{\epsilon}_{t}, \\
z_{t} & =\tilde{D}_{t} \tilde{u}_{t},
\end{aligned}
$$

where $\tilde{u}_{t}=\bar{T}^{-1} u_{t}, \tilde{\epsilon}_{t}=\bar{T}^{-1} \epsilon_{t}$ and $\tilde{D}_{t}=\bar{D}_{t} \bar{T}$.
Lemma 4 Let $A=T J T^{-1}$ be the Jordan normal form of $A$. Then

$$
\bar{J}=\operatorname{diag}\left(J, Z_{1}, \cdots, Z_{L}\right),
$$

where $Z_{l}, l \in\{1, \cdots, L\}$ are $m$-dimensional Jordan blocks with zero eigenvalues, and there exist matrices $U$ and $V$ such that

$$
\bar{T}=\left[\begin{array}{cc}
T & 0 \\
U & V
\end{array}\right] .
$$

PROOF. Let $\bar{t}=\left[0, v^{\top}\right]^{\top}$ with $v^{\top}=\left[v_{1}^{\top}, \cdots, v_{L}^{\top}\right]$. We then have

$$
\bar{A} \bar{t}=\bar{A}\left[0, v^{\top}\right]^{\top}=\left[0,0, v_{1}^{\top}, \cdots, v_{L-1}^{\top}\right]^{\top} .
$$

It follows that $\bar{A}$ has $m$ generalized eigenvectors of rank $L$ with zero associated eigenvalue. Now, suppose that $t$ is an eigenvector of $A$ with eigenvalue $\lambda$. Let

$$
\bar{t}=\left[t^{\top},(C t)^{\top}, \lambda^{-1}(C t)^{\top}, \ldots, \lambda^{-L+2}(C t)^{\top}\right]^{\top}
$$

It is straightforward to show that $\bar{A} \bar{t}=\lambda \bar{t}$. Hence, $\bar{t}$ is an eigenvector of $\bar{A}$ with eigenvalue $\lambda$. Using a similar but somehow more tedious argument, we can show that, for any generalized eigenvector $t$ of $A$, there will be a generalized eigenvector $\bar{t}$ of $\bar{A}$, with the same order and eigenvalue. Hence, the whole set of generalized eigenvectors of $\bar{A}$ is formed by either $\bar{t}=\left[0, v^{\top}\right]^{\top}$ for some $v$ or $\bar{t}=\left[t^{\top}, u^{\top}\right]^{\top}$, for some $u$ and $t$ being a generalized eigenvector of $A$. Thus, (9) follows. Also, the first $n$ eigenvalues of $\bar{A}$ equal those of $A$, and the remaining are all zero. Hence, (8) follows.

Definition $5 A$ set of complex numbers $x_{i} \in \mathbb{C}, i=$ $1, \cdots, I$, is said to have a common finite multiplicative order $\iota \in \mathbb{N}$ up to a constant $a \in \mathbb{C}$, if $x_{i}^{\iota}=a^{\iota}$, for all $i=$ $1, \cdots, I$. If there do not exist $\iota$ and a satisfying the above, the set is said not to have common finite multiplicative order 2

It is straightforward to see that there is a unique partition of $J$ in diagonal blocks of the form

$$
J=\operatorname{diag}\left(J_{1}, \cdots, J_{K}\right),
$$

such that, for every $k=1, \ldots, K$, the diagonal entries of the sub-matrices $J_{k}$ have a common finite multiplicative order $\iota_{k}$ up to $a_{k}$, and for any $k \neq l$, the diagonal entries

2 For example, $e^{\frac{\pi}{2} \jmath}$ and $e^{\pi \jmath}$ have a common finite multiplicative order for that $\left(e^{\frac{\pi}{2} \jmath}\right)^{4}=\left(e^{\pi_{J}}\right)^{4}=1$. While, $e^{\frac{\pi}{\sqrt{2} J}}$ and $e^{\pi_{J}}$ do not have a common finite multiplicative order since there does not exist $k \in \mathbb{N}$ such that $\left(e^{\frac{\pi}{\sqrt{2}} \jmath}\right)^{k}=\left(e^{\pi \jmath}\right)^{k}=1$. 
of the matrix $\operatorname{diag}\left(J_{k}, J_{l}\right)$ do not have common finite multiplicative order. Let $d_{k}$ denote the dimension of $J_{k}$ and $a_{k}$ its magnitude. For each $t \in \mathbb{N}$, let

$$
\tilde{D}_{t}=\left[\tilde{D}_{t, 1}, \cdots, \tilde{D}_{t, K}, \tilde{D}_{t, *}\right],
$$

be the partition of $\tilde{D}_{t}$ defined such that, for every $k=1, \ldots, K$, the number of columns of $\tilde{D}_{t, k}$ equals the dimension of $J_{k}$. Let

$$
O_{t, T, k}=\left[\begin{array}{c}
\tilde{D}_{t, k} J_{k}^{t} \\
\vdots \\
\tilde{D}_{t+T-1, k} J_{k}^{t+T-1}
\end{array}\right]
$$

Our next step is to state a necessary and sufficient condition for the stability of (6)-(7). To this end, we aim to use the result in [15, Theorem 14]. This result is stated under assumptions which are very general, but technically involved. Fortunately, we have a way around this technical difficulty. We have that $\left\{\tilde{D}_{t}: t \in \mathbb{N}\right\}$ is a sequence of random matrices, with discrete distribution, such that $\left\{\tilde{D}_{t}: t \in \mathbb{N}\right\}$ is a statistically independent set, and whose statistics are cyclostationary with period $M$. Hence, it follows from [15, Proposition 18] that the conditions for [15, Theorem 14] are guaranteed. Also, these conditions consider all FMO blocks of (6)-(7). In view of Lemma 4, this system has $K+1$ blocks. However, the eigenvalue of the last FMO block equals zero. Hence, the conditions need only consider the first $K$ blocks. We then obtain the following result.

Theorem 6 (Combination of [15, Proposition 18] and [15, Theorem 14]) Suppose that the sequence of coding matrices $\left\{H_{t}: t \in \mathbb{N}\right\}$ is $P$-periodic, i.e., $H_{t+P}=H_{t}$, for all $t \in \mathbb{N}$. Then, the MMSE estimator using coded measurements is stable if

$$
\max _{1 \leq k \leq K}\left|a_{k}\right|^{2} \Phi_{k}<1
$$

and unstable if

$$
\max _{1 \leq k \leq K}\left|a_{k}\right|^{2} \Phi_{k}>1
$$

where the channel unreliability measure $\Phi_{k}$ with respect to block $k$ is defined by

$$
\Phi_{k}=\max _{0 \leq t<M} \limsup _{T \rightarrow \infty} \mathbb{P}\left(O_{t, T, k} \text { does not have } F C R\right)^{1 / T}
$$

and $M$ is the least common multiple of $P$ and $\iota_{k}, k \in$ $\{1, \cdots, K\}$.

Remark 7 The result above is inconclusive for the case of $\max _{1 \leq k \leq K}\left|a_{k}\right|^{2} \Phi_{k}=1$. For this reason, we say that the necessary and sufficient condition in Theorem 6 has a trivial gap.

In order to evaluate the condition in Theorem 6 , we need to compute the channel unreliability measure $\Phi_{k}$ with respect to each block $k$. This measure depends on the design parameters $L$ and $H_{t}, t \in \mathbb{N}$. Our next goal is to provide an expression of $\Phi_{k}$, together with the choices of $L$ and $H_{t}, t \in \mathbb{N}$, so that we can minimize $\Phi_{k}$.

The measure $\Phi_{k}$ is defined in terms of the probability that the matrix $O_{t, T, k}$ does not have full column rank (FCR). Our first step towards the computation of $\Phi_{k}$ is to replace $O_{t, T, k}$ by a different matrix, which we denote by $\tilde{O}_{t, T, k}$, for which the aforementioned probability is easier to compute.

For each $i \in\{1, \cdots, I\}$, let $\tilde{C}^{i}=C^{i} T$ and

$$
\tilde{C}^{i}=\left[\tilde{C}_{1}^{i}, \cdots, \tilde{C}_{K}^{i}\right]
$$

be the partition of $\tilde{C}^{i}$ defined such that, for every $k=$ $1, \ldots, K$, the number of columns of $\tilde{C}_{k}^{i}$ equals the dimension of $J_{k}$. Let $H_{t}^{i}=\left[H_{t, 1}^{i}, \cdots, H_{t, L}^{i}\right]$ and define, for each $k \in\{1, \cdots, K\}$ and $t, T \in \mathbb{N}$, the following matrix

$\begin{aligned} \tilde{O}_{t, T, k} & =\left[\begin{array}{c}\tilde{O}_{t, T, k}^{1} \\ \vdots \\ \tilde{O}_{t, T, k}^{I}\end{array}\right], \quad \text { with } \quad \tilde{O}_{t, T, k}^{i}=\left[\begin{array}{c}\tilde{o}_{k, t}^{i} \\ \vdots \\ \tilde{o}_{k, t+T-1}^{i}\end{array}\right], \\ \tilde{o}_{k, t}^{i}=\Gamma_{t}^{i} H_{t}^{i} G_{k}^{i} J_{k}^{t}, & \text { and } \quad G_{k}^{i}=\left[\begin{array}{c}\tilde{C}_{k}^{i} \\ \vdots \\ \tilde{C}_{k}^{i} J_{k}^{-L+1}\end{array}\right] .\end{aligned}$

We have the following result.

Lemma 8 For each $k \in\{1, \cdots, K\}$,

$\Phi_{k}=\max _{0 \leq t<M} \limsup _{T \rightarrow \infty} \mathbb{P}\left(\tilde{O}_{t, T, k} \text { does not have } F C R\right)^{1 / T}$.

PROOF. See Appendix A.

Our next step is to use Lemma 8 to provide an expression for $\Phi_{k}$, together with the choices of $L$ and $H_{t}, t \in \mathbb{N}$, minimizing its value. 
Recall that the packet arrival at time $t$ is represented by the diagonal matrix $\Gamma_{t}$. Let $\mathbb{D}$ denote the set of all possible values of $\Gamma_{t}$. We use $\Gamma_{t, T}=\left\{\Gamma_{t}, \cdots, \Gamma_{t+T-1}\right\} \in \mathbb{D}^{T}$ to represent the packet arrivals in the past-time horizon of length $T$ starting from $t$. For given packet-arrival pattern $S \in \mathbb{D}^{M}$, we use $\nu^{i}(S)$ to denote the number of measurements from node $i$ included in $S$.

Let $\mathcal{G}_{k}^{i}$ denote the row span of

$$
\Omega_{k}^{i}=\left[\begin{array}{c}
G_{k}^{i} \\
\vdots \\
G_{k}^{i} J_{k}^{d_{k}-1}
\end{array}\right]
$$

Definition 9 We say that set $\mathcal{L} \subseteq\{1, \cdots, I\}$ of nodes is insufficient for block $k$ if

$$
\operatorname{span}\left(\bigcup_{i \in \mathcal{L}} \mathcal{G}_{k}^{i}\right) \neq \mathbb{R}^{d_{k}}
$$

An insufficient set $\mathcal{L}$ is maximal if either $\mathcal{L}=\{1, \cdots, I\}$ or, for all $i \notin \mathcal{L}$, the set $\mathcal{L} \cup\{i\}$ is not insufficient. Let $\mathbb{L}_{k}$ denote the collection of all maximal insufficient sets for block $k$.

Recall that $c_{i}$ is the dimension of $z_{k}^{i}$. For each $\mathcal{L} \in$ $\mathbb{L}_{k}$, we say that a set of measurement counts $\mathcal{M}=$ $\left\{0 \leq M^{i} \leq M: i \notin \mathcal{L}\right\}$ is insufficient for $\mathcal{L}$ and $k$ if, for any choice of $c_{i} M^{i}$ vectors $v_{j}^{i} \in \mathcal{G}_{k}^{i}, i \notin \mathcal{L}, j \in$ $\left\{1, \cdots, c_{i} M^{i}\right\}$, we have

$$
\operatorname{span}\left(\bigcup_{i \in \mathcal{L}} \mathcal{G}_{k}^{i} \cup \bigcup_{\substack{i \neq \mathcal{L} \\ j \in\left\{1, \cdots, c_{i} M^{i}\right\}}} v_{j}^{i}\right) \neq \mathbb{R}^{d_{k}}
$$

An insufficient set of counts $\mathcal{M}=$ $\left\{0 \leq M^{i} \leq M: i \notin \mathcal{L}\right\}$ is maximal if for any $i \notin \mathcal{L}$ for which $M^{i}<M$, the set obtained by replacing $M^{i}$ by $M^{i}+1$ is not insufficient. Let $\mathbb{M}_{k}(\mathcal{L})$ denote the collection of all maximal insufficient sets of counts for $\mathcal{L}$ and $k$. Recall that $p_{i}=\mathbb{E}\left[\gamma_{t}^{i}\right], i \in\{1, \cdots, I\}$, denotes the packet receival rate for sensor $i$. We have the following result.

Assumption 10 The sequence of coding matrices $\left\{H_{t}\right.$ : $t \in \mathbb{N}\}$ is $P$-periodic and generated using a pseudorandom sequence with absolutely continuous distribution.

Theorem 11 Under Assumption 10, if $L \geq d_{k}$ for all $k \in\{1, \cdots, K\}$, then w.p.1 over the random outcomes of $H_{t}$, the resulting value of $\Phi_{k}$ is minimized w.r.t. $L$ and
$H_{t}, t \in \mathbb{N}$. Furthermore, its value is

$$
\Phi_{k}=\max _{\mathcal{L} \in \mathbb{L}_{k}} \max _{\mathcal{M} \in \mathbb{M}_{k}(\mathcal{L})} \prod_{i \notin \mathcal{L}}\left(1-p_{i}\right)^{1-\frac{M^{i}}{M}}
$$

where $M$ is the least common multiple of $P$ and $\iota_{k}$ for any $k \in\{1, \cdots, K\}$.

PROOF. See Appendix B.

Remark 12 We point out that, while Theorem 11 asserts that the coding matrix design the stated in Assumption 10 is optimal for the purpose of estimator stability, the same design may not be optimal for the purpose of minimizing the estimation error covariance.

Remark 13 For a given FMO block $k \in\{1, \cdots, K\}$, maximal insufficient set $\mathcal{L} \in \mathbb{L}_{k}$ and node $i \notin \mathcal{L}$, the vectors $v_{j}^{i}, j \in\left\{1, \cdots, c_{i} M^{i}\right\}$ belong to the subspace $\mathcal{G}_{k}^{i} \subset \mathbb{R}^{d_{k}}$. Suppose that the FMO block $k$ is observable, i.e., $\operatorname{span}\left(\bigcup_{i=1}^{I} \mathcal{G}_{k}^{i}\right)=\mathbb{R}^{d_{k}}$. Let $\mathcal{M}=$ $\left\{0 \leq M^{i} \leq M: i \notin \mathcal{L}\right\} \in \mathbb{M}_{k}(\mathcal{L})$ be a maximal insufficient set of counts for $\mathcal{L}$. Then, since (13) needs to hold for any choice of $v_{j}^{i}$ 's, it follows that an increment in the dimension $c_{i}$ of the data transmitted by sensor $i$ would lead to a reduction of the measurement count $M^{i}$ for that sensor. In view of 14 , this will in turn reduce $\Phi_{k}$. Hence, there is a tradeoff between the communication load (i.e., the value of $c_{i}$ for all $i \in\{1, \cdots, I\}$ ) and the robustness to packet losses (i.e., the value of $\Phi_{k}$ ).

The above expression of $\Phi_{k}$ greatly simplifies in the limit case as the period $P$ of the pseudo-random sequence used to generate $H_{t}, t \in \mathbb{N}$ tends to infinity. This is stated in the following corollary of Theorem 11. This result represents most practical situations, as periods of pseudo-random sequences are typically very large.

Corollary 14 Under the assumptions of Theorem 11,

$$
\lim _{P \rightarrow \infty} \Phi_{k}=\max _{\mathcal{L} \in \mathbb{L}_{k}} \prod_{i \notin \mathcal{L}}\left(1-p_{i}\right) .
$$

PROOF. Notice that, in view of the choices of $L$ and $H_{t}$, if $\operatorname{rank}\left(\tilde{O}_{t, T, k}^{i}\right)<\operatorname{rank}\left(G_{k}^{i}\right)$, then every new measurement from node $i$ yields $\operatorname{rank}\left(\tilde{O}_{t, T+1, k}^{i}\right) \geq$ $\operatorname{rank}\left(\tilde{O}_{t, T, k}^{i}\right)+1$. Hence, for any $\mathcal{L} \in \mathbb{L}_{k}$ and $\mathcal{M} \in$ $\mathbb{M}_{k}(\mathcal{L})$, we must have that $M^{i}<\operatorname{rank}\left(G_{k}^{i}\right)$, for all $i \notin \mathcal{L}$. 
Since $M$ tends to infinity as so does $P$, we have

$$
\begin{aligned}
\lim _{P \rightarrow \infty} \Phi_{k} & =\lim _{P \rightarrow \infty} \max _{\mathcal{L} \in \mathbb{L}_{k}} \max _{\mathcal{M} \in \mathbb{M}_{k}(\mathcal{L})} \prod_{i \notin \mathcal{L}}\left(1-p_{i}\right)^{1-\frac{M^{i}}{M}} \\
& =\max _{\mathcal{L} \in \mathbb{L}_{k}} \max _{\mathcal{M} \in \mathbb{M}_{k}(\mathcal{L})} \prod_{i \notin \mathcal{L}}\left(1-p_{i}\right) \\
& =\max _{\mathcal{L} \in \mathbb{L}_{k}} \prod_{i \notin \mathcal{L}}\left(1-p_{i}\right)
\end{aligned}
$$

Remark 15 The above Corollary 14 shows that, when the period $P$ of the pseudo-random sequence used to generate coding matrices is sufficiently large, the stability condition is no longer affected by the dimension $c_{i}$ of coded measurements. Nevertheless, a larger value of $c_{i}$ is still helpful to improve the accuracy of the estimation.

\subsection{An easily verifiable sufficient condition}

The necessary and sufficient condition stated in Theorem 11 requires splitting the system in $K$ blocks. In this section we derive a condition which is only sufficient, but simpler to compute as it does not require the aforementioned splitting.

Let

$$
\Xi_{k}=\left[\begin{array}{c}
\Gamma_{(k-1) P} H_{(k-1) P} F \\
\vdots \\
\Gamma_{k P-1} H_{k P-1} F A^{P-1}
\end{array}\right]
$$

with

$$
F=\left[\begin{array}{c}
C \\
\vdots \\
C A^{L-1}
\end{array}\right]
$$

We have the following result.

Lemma 16 If $\Xi_{k}$ does not have full column rank,

$$
P_{k P \mid k P-1} \leq \rho(A)^{2 P} \frac{\rho(A)^{2}}{\rho(A)^{2}-1} P_{(k-1) P \mid(k-1) P-1},
$$

where $\rho(A)$ denotes the spectral radius of $A$. If $\Xi_{k}$ has full column rank, there exists $0<\bar{P} \in \mathbb{R}^{n \times n}$ independent of $P_{(k-1) P \mid(k-1) P-1}$, such that

$$
P_{k P \mid k P-1} \leq \bar{P} .
$$

PROOF. See Appendix C.
For each $i \in\{1, \cdots, I\}$, let

$$
F^{i}=\left[\begin{array}{c}
C^{i} \\
\vdots \\
C^{i} A^{L-1}
\end{array}\right]
$$

Definition 17 A set $\mathcal{Q}=\left\{q^{i} \in \mathbb{N}: i=1, \cdots, I\right\}$ of integers is called feasible if, for each $i \in\{1, \cdots, I\}$, there exist $q^{i}$ indexes $r_{j}^{i}, j \in\left\{1, \cdots, q^{i}\right\}$, such that the matrix

$$
\operatorname{span}\left(\bigcup_{i=1}^{I} \bigcup_{j=1}^{q^{i}} \operatorname{row}_{r_{j}^{i}}\left(F^{i}\right)\right)=\mathbb{R}^{n}
$$

where $\operatorname{row}_{r}(X)$ denotes the vector formed by the $r$-th row of matrix $X$. We use $\mathbb{Q}$ to denote the collection of all feasible sets.

We now state the main result of this subsection.

Theorem 18 Under Assumption 10, if $P, L \geq n$, then, w.p. 1 over the random outcomes of $H_{t}$, the MMSE estimator using coded measurements is stable if

$$
|\rho(A)|^{2} \pi_{P}^{1 / P}<1
$$

where

$$
\pi_{P}=\left(\frac{\rho(A)^{2}}{\rho(A)^{2}-1}\right) \sum_{\mathcal{Q} \notin \mathbb{Q}} \prod_{i=1}^{I}\left(\begin{array}{l}
P \\
q^{i}
\end{array}\right) p_{i}^{q_{i}}\left(1-p_{i}\right)^{P-q_{i}},
$$

with $\left(\begin{array}{c}P \\ q^{i}\end{array}\right)$ denoting the binomial coefficient $P$ choose $q^{i}$. Moreover, if $\left(A, C^{i}\right)$ is observable for $i=1, \cdots, I$, then the estimator is unstable if

$$
\lim _{P \rightarrow \infty}|\rho(A)|^{2} \pi_{P}^{1 / P}>1 .
$$

PROOF. See Appendix D.

\section{State estimation comparison using raw and coded measurements}

In this section we derive the stability condition using raw measurements to compare with that using coded measurements.

Consider the system described in Section 2. Suppose that the raw measurements $y_{t}$, as opposite to the coded ones $z_{t}$, are transmitted to the estimator, using the same channel described in Section 2. The MMSE estimator 
then becomes a Kalman filter, having the following information available at time $t$ :

$$
\breve{\mathcal{F}}_{t}=\left\{\left(\Gamma_{1}, \Gamma_{1} y_{1}\right), \cdots,\left(\Gamma_{t}, \Gamma_{t} y_{t}\right)\right\}
$$

Recall that, for block $k, \iota_{k}$ denotes its finite multiplicative order and $\mathbb{L}_{k}$ denotes the collection of all maximal insufficient sets. For $\mathcal{L} \in \mathbb{L}_{k}$, we say that a set $\mathcal{I}(\mathcal{L})=\left\{0 \leq q_{j}^{i}<\iota_{k}: i \notin \mathcal{L}, j=1, \cdots, \check{M}_{i}\right\}$ of indexes is insufficient for block $k$ if

$$
\breve{\Omega}_{k}=\left[\begin{array}{c}
\breve{\Omega}_{k}^{1} \\
\vdots \\
\breve{\Omega}_{k}^{I}
\end{array}\right] \text { does not have full column rank, }
$$

where,

$$
\breve{\Omega}_{k}^{i}=\left[\begin{array}{c}
\tilde{C}_{k}^{i} \\
\vdots \\
\tilde{C}_{k}^{i} J_{k}^{\iota_{k}}
\end{array}\right] \text { if } i \in \mathcal{L} \text { and }\left[\begin{array}{c}
\tilde{C}_{k}^{i} J_{k}^{q_{1}^{i}} \\
\vdots \\
\tilde{C}_{k}^{i} J_{k}^{q_{\check{M}_{i}}^{i}}
\end{array}\right] \text { otherwise }
$$

with $\tilde{C}_{k}^{i}$ defined in (11). We say that an insufficient set of index is maximal if the set obtained by adding any extra index is not insufficient. Let $\mathbb{I}_{k}(\mathcal{L})$ denote the collection of maximal insufficient index sets for block $k$ and set $\mathcal{L}$. For $\mathcal{I} \in \mathbb{I}_{k}(\mathcal{L})$ we use $\nu^{i}(\mathcal{I})$ to denote the number of indexes from node $i$ included in $\mathcal{I}$.

The following result then states the desired stability condition.

Proposition 19 The MMSE estimator using raw measurements is stable if

$$
\max _{1 \leq k \leq K}\left|a_{k}\right|^{2} \breve{\Phi}_{k}<1
$$

and unstable if

$$
\max _{1 \leq k \leq K}\left|a_{k}\right|^{2} \breve{\Phi}_{k}>1
$$

where

$$
\breve{\Phi}_{k}=\max _{\mathcal{L} \in \mathbb{L}_{k}} \max _{\mathcal{I} \in \mathbb{I}_{k}(\mathcal{L})} \prod_{i \notin \mathcal{L}}\left(1-p_{i}\right)^{1-\frac{\nu^{i}(\mathcal{I})}{\iota_{k}}} .
$$

PROOF. Using Theorem 6, the result holds with

$$
\breve{\Phi}_{k}=\max _{0 \leq t<\iota_{k}} \limsup _{T \rightarrow \infty} \mathbb{P}\left(\breve{O}_{t, T, k} \text { does not have FCR }\right)^{1 / T}
$$

where

$$
\breve{O}_{t, T, k}=\left[\begin{array}{c}
\left(\Gamma_{t} \otimes I_{m}\right) \tilde{C}_{k} \\
\vdots \\
\left(\Gamma_{T-1} \otimes I_{m}\right) \tilde{C}_{k} J_{k}^{T-1}
\end{array}\right]
$$

with $\tilde{C}_{k}^{\top}=\left[\tilde{C}_{k}^{1 \top}, \cdots, \tilde{C}_{k}^{I \top}\right]$. For $S \in \mathbb{D}^{T}$ we use $\breve{O}_{t, T, k}(S)$ to denote the value of $\breve{O}_{t, T, k}$ resulting when $\Gamma_{t, T}=S$.

Let $\mathbb{S}_{k} \subset \mathbb{D}^{\iota_{k}}$ be the set of all $S \in \mathbb{D}^{\iota_{k}}$ such that $\breve{O}_{t, \iota_{k}, k}(S)$ does not have full column rank. We can then use Lemma 21 to obtain

$$
\breve{\Phi}_{k}=\max _{S \in \mathbb{S}_{k}} \varsigma_{k}(S)^{1 / \iota_{k}}
$$

where

$$
\varsigma_{k}(S)=\mathbb{P}\left\{\operatorname{ker}\left(\breve{O}_{0, \iota_{k}, k}\left(\Gamma_{0, \iota_{k}}\right)\right) \supseteq \operatorname{ker}\left(\breve{O}_{0, \iota_{k}, k}(S)\right)\right\} .
$$

The result then follows after noticing that, if $S_{1}$ contains the measurements in all entries where $S_{2}$ also does, then $\varsigma_{k}\left(S_{1}\right) \geq \varsigma_{k}\left(S_{2}\right)$.

We now compare the stability conditions resulting from using coded and raw measurements. To this end, for the coded case, since the period $P$ of pseudo-random measurements is typically very large, we use the asymptotic result given in Corollary 14.

Let $\mathcal{L}_{\mathrm{c}}$ denote the argument which maximizes (15). We have

$$
\lim _{P \rightarrow \infty} \Phi_{k}=\prod_{i \notin \mathcal{L}_{\mathrm{c}}}\left(1-p_{i}\right)
$$

Let also $\mathcal{L}_{\mathrm{r}}$ be the one maximizing (20). We then have

$$
\begin{aligned}
\breve{\Phi}_{k} & =\max _{\mathcal{I} \in \mathbb{I}_{k}\left(\mathcal{L}_{\mathrm{r}}\right)} \prod_{i \notin \mathcal{L}_{\mathrm{r}}}\left(1-p_{i}\right)^{1-\frac{\nu^{i}(\mathcal{I})}{\iota_{k}}} \\
& \geq \max _{\mathcal{I} \in \mathbb{I}_{k}\left(\mathcal{L}_{\mathrm{c}}\right)} \prod_{i \notin \mathcal{L}_{\mathrm{c}}}\left(1-p_{i}\right)^{1-\frac{\nu^{i}(\mathcal{I})}{\iota_{k}}} \\
& =\prod_{i \notin \mathcal{L}_{\mathrm{c}}}\left(1-p_{i}\right) \times \max _{\mathcal{I} \in \mathbb{I}_{k}\left(\mathcal{L}_{\mathrm{c}}\right)} \prod_{i \notin \mathcal{L}_{\mathrm{c}}}\left(1-p_{i}\right)^{-\frac{\nu^{i}(\mathcal{I})}{\iota_{k}}} .
\end{aligned}
$$

We then obtain

$$
\begin{aligned}
\frac{\lim _{P \rightarrow \infty} \Phi_{k}}{\breve{\Phi}_{k}} & \leq\left(\max _{\mathcal{I} \in \mathbb{I}_{k}\left(\mathcal{L}_{\mathrm{c}}\right)} \prod_{i \notin \mathcal{L}_{\mathrm{c}}}\left(1-p_{i}\right)^{-\frac{\nu^{i}(\mathcal{I})}{\iota_{k}}}\right)^{-1} \\
& =\max _{\mathcal{I} \in \mathbb{I}_{k}\left(\mathcal{L}_{\mathrm{c}}\right)} \prod_{i \notin \mathcal{L}_{\mathrm{c}}}\left(1-p_{i}\right)^{\frac{\nu^{i}(\mathcal{I})}{\iota_{k}}} \\
& <1
\end{aligned}
$$


which clearly shows the stability improvement offered by the proposed coding scheme.

\section{Example}

In this section we use an example to illustrate the improvement, in terms of the stability of the MMSE estimator, given by the proposed coding scheme. To this end, we compare the stability of the estimator in the cases of raw and coded measurements.

We use a system as described in Section 2, with

$$
\begin{aligned}
A & =\left[\begin{array}{cccc}
2 & -4 & -4.5 & 3 \\
0 & -2 & -3.5 & 3 \\
0 & 0 & 1.5 & 0 \\
0 & 0 & 0 & 1
\end{array}\right] \\
& =\underbrace{\left[\begin{array}{llll}
1 & 1 & 1 & 1 \\
0 & 1 & -1 & 1 \\
0 & 0 & 1 & 0 \\
0 & 0 & 0 & 1
\end{array}\right]}_{T} \underbrace{\left[\begin{array}{cccc}
2 & 0 & 0 & 0 \\
0 & -2 & 0 & 0 \\
0 & 0 & 1.5 & 0 \\
0 & 0 & 0 & 1
\end{array}\right]}_{J} \underbrace{\left[\begin{array}{cccc}
1 & -1 & -2 & 0 \\
0 & 1 & 1 & -1 \\
0 & 0 & 1 & 0 \\
0 & 0 & 0 & 1
\end{array}\right]}_{T^{-1}}
\end{aligned}
$$

and $Q=\operatorname{diag}\{1,1,1,1\}$. There are $\mathrm{I}=4$ sensors, with $C^{1}=\left[\begin{array}{llll}1 & 1 & 1 & 1\end{array}\right], C^{2}=\left[\begin{array}{llll}1 & -1 & 1 & 1\end{array}\right], C^{3}=\left[\begin{array}{llll}1 & 1 & 1 & -1\end{array}\right]$, $C^{4}=\left[\begin{array}{llll}1 & -1 & 1 & -1\end{array}\right]$ and $R^{1}=R^{2}=R^{3}=R^{4}=1$.

Also, the communication channels used to transmit sensor measurements, either raw or coded ones, have packet receival rates $p_{1}=p_{2}=p_{3}=p_{4}=0.4$.

For the coding parameters, we let $L=4$ and $P=500$. For all $t \in\{1, \cdots, P\}, l \in\{1, \cdots, L\}$ and $i \in\{1, \cdots, I\}$, we randomly generate the entries of $H_{t, l}^{i}$ by drawing them from a standard normal distribution. Since the period $P=500$ is much larger than the state dimension $n=4$, we assess the stability of the MMSE estimator using Corollary 14

It follows from (22) that the system is formed by $K=3$ FMO blocks. The magnitude of these blocks are $a_{1}=2$, $a_{2}=1.5$ and $a_{3}=1$, and their finite multiplicative orders are $\iota_{1}=2, \iota_{2}=1$ and $\iota_{3}=1$. Also, the blocks are observable from all nodes, hence $\mathbb{L}_{1}=\mathbb{L}_{2}=\mathbb{L}_{3}=\emptyset$. We then have

$$
\Phi_{1}=\Phi_{2}=\Phi_{3}=\prod_{i=1}^{4}\left(1-p_{i}\right)=(1-0.4)^{4}=0.1296
$$

Hence, the filter is stable since

$$
\left|a_{3}\right|^{2} \Phi_{3}<\left|a_{2}\right|^{2} \Phi_{2}<\left|a_{1}\right|^{2} \Phi_{1}=2^{2} \times 0.1296<1 .
$$

Since $\left(A, C^{i}\right)$ is observable, for all $i=1,2,3$, we have that the sufficient condition in Theorem 18 is equivalent to the necessary and sufficient one given in Theorem 11. Then, since $P$ is large, we have

$$
\left(\pi_{P}\right)^{1 / P} \simeq 0.1296
$$

For the uncoded case, we start by analyzing the first FMO block. We have

$$
\begin{gathered}
\tilde{C}_{1}^{1}=\tilde{C}_{1}^{3}=[1,2], \quad \tilde{C}_{1}^{2}=\tilde{C}_{1}^{4}=[1,0], \\
\tilde{C}_{1}^{1} J_{1}=\tilde{C}_{1}^{3} J_{1}=[2,-4], \tilde{C}_{1}^{2} J_{1}=\tilde{C}_{1}^{4} J_{1}=[2,0] .
\end{gathered}
$$

Hence, the collection $\mathbb{I}_{1}(\emptyset)=\left\{\mathcal{I}_{1,1}, \mathcal{I}_{1,2}, \mathcal{I}_{1,3}\right\}$ of maximal insufficient index sets contains three sets. Set $\mathcal{I}_{1,1}$ contains index 0 from node 1 and index 0 from node 3 , the set $\mathcal{I}_{1,2}$ contains index 1 from node 1 and index 1 from node 3 and set $\mathcal{I}_{1,3}$ contains indexes 0 and 1 from nodes 2 and 4 . From Theorem 19, we have

$$
\breve{\Phi}_{1}=\max _{\mathcal{I} \in \mathbb{I}_{1}(\emptyset)} \prod_{i=1}^{4}\left(1-p_{i}\right)^{1-\frac{\nu^{i}(\mathcal{I})}{{ }^{\prime} 1}} .
$$

Now,

$$
\begin{aligned}
& \prod_{i=1}^{4}\left(1-p_{i}\right)^{1-\frac{\nu^{i}\left(\mathcal{I}_{1,1}\right)}{\iota_{1}}}=0.216, \\
& \prod_{i=1}^{4}\left(1-p_{i}\right)^{1-\frac{\nu^{i}\left(\mathcal{I}_{1,2}\right)}{\iota_{2}}}=0.216, \\
& \prod_{i=1}^{4}\left(1-p_{i}\right)^{1-\frac{\nu^{i}\left(\mathcal{I}_{1,3}\right)}{\iota_{2}}}=0.36 .
\end{aligned}
$$

Hence, $\breve{\Phi}_{1}=0.36$, and for the first FMO block we obtain

$$
\left|a_{1}\right|^{2} \breve{\Phi}_{1}=2^{2} \times 0.36>1
$$

We therefore do not need to evaluate $\breve{\Phi}_{2}$ and $\breve{\Phi}_{3}$, since the above inequality in enough to assert that the estimator is unstable.

The above claims are illustrated in Figure 3. The figure shows the time evolution of the norm of the prediction error covariance yield by the MMSE estimator using both, raw and coded measurements. To this end we average over $2 \times 10^{4}$ Monte Carlo runs. The figure clearly shows that the MMSE estimator is stable when using coded measurements, while unstable when using raw ones.

\section{Conclusion}

We studied the networked MMSE state estimation problem for a linear system with a distributed set of sensors. 


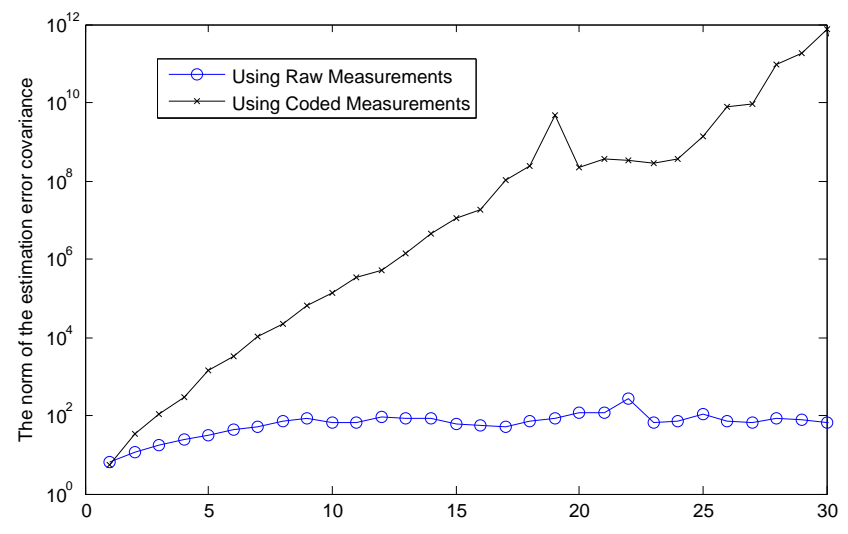

Fig. 3. Norm of prediction error covariance using coded and raw measurements.

We proposed a measurement coding scheme which permits both, controlling the load of communication used for estimation, and maximizing, within the family of linear causal coders, the robustness of the resulting estimator against packet losses. We derived the resulting MMSE estimator, and state a necessary and sufficient condition, having a trivial gap, for its stability. We quantified the robustness gain offered by the proposed scheme, by comparing the stability condition to the one resulting from the use of raw measurements. We presented simulation results to confirm our claims.

\section{A Proofs of Lemma 8}

Notation 20 Let $\mathcal{F C R}$ denote the set of matrices having full column rank.

Let

$$
\bar{T}=\left[\bar{T}_{1}, \cdots, \bar{T}_{K}, \bar{T}_{*}\right],
$$

be the partition of $\bar{T}$ defined such that, for every $k$, the number of columns of $\bar{T}_{k}$ equals the dimension of $J_{k}$. We have

$$
\tilde{D}_{t, k} J_{k}^{t}=\bar{D}_{t} \bar{T}_{k} J_{k}^{t}
$$

Now $\bar{A} \bar{T}=\bar{T} \bar{J}$. Hence, from (8),

$$
\bar{A} \bar{T}_{k}=\bar{T} \operatorname{diag}\left(0, J_{k}, 0\right)=\bar{T}_{k} J_{k} .
$$

Putting the above into (A.1) we obtain

$$
\tilde{D}_{t, k} J_{k}^{t}=\bar{D}_{t} \bar{A}^{t} \bar{T}_{k}
$$

Now, it is straightforward to see that

$$
\bar{A}^{t}=\left[\begin{array}{cc}
A^{t} & 0 \\
R_{t} & Q^{t}
\end{array}\right], \quad \text { with } \quad Q=\left[\begin{array}{ll}
0 & 0 \\
I & 0
\end{array}\right],
$$

$R_{0}=0$ and, for $t>0$,

$$
R_{t}=R_{t-1} A+Q^{t-1} R .
$$

Putting (A.3) into (A.2), and recalling (9), we obtain

$$
\begin{aligned}
\tilde{D}_{t, k} J_{k}^{t} & =\Gamma_{t}\left[\begin{array}{ll}
0 & H_{t}
\end{array}\right]\left[\begin{array}{cc}
A^{t} & 0 \\
R_{t} & Q^{t}
\end{array}\right]\left[\begin{array}{l}
T_{k} \\
U_{k}
\end{array}\right] \\
& =\Gamma_{t} H_{t}\left[\begin{array}{ll}
R_{t} & Q^{t}
\end{array}\right]\left[\begin{array}{c}
T_{k} \\
U_{k}
\end{array}\right] \\
& =\Gamma_{t} H_{t} R_{t} T_{k}+\Gamma_{t} H_{t} Q^{t} U_{k} .
\end{aligned}
$$

Now, for $t \geq L$, we have

$$
R_{t}=\left[\begin{array}{c}
C A^{t} \\
\vdots \\
C A^{t-L+1}
\end{array}\right] .
$$

We then obtain

$$
\begin{aligned}
& \Gamma_{t} H_{t} R_{t} T_{k} \\
= & \Gamma_{t} H_{t}\left[\begin{array}{c}
C A^{t} \\
\vdots \\
C A^{t-L+1}
\end{array}\right] T_{k}=\Gamma_{t} H_{t}\left[\begin{array}{c}
C T_{k} J_{k}^{t} \\
\vdots \\
C T_{k} J_{k}^{t-L+1}
\end{array}\right] \\
= & \Gamma_{t} H_{t}\left[\begin{array}{c}
\tilde{C}_{k} \\
\vdots \\
\tilde{C}_{k} J_{k}^{-L+1}
\end{array}\right] J_{k}^{t}=\left[\begin{array}{c}
\tilde{o}_{k, t}^{1} \\
\vdots \\
\tilde{o}_{k, t}^{I}
\end{array}\right] \\
\triangleq & \tilde{O}_{t, k} .
\end{aligned}
$$

For $t \geq L$ we also have $Q^{t}=0$, and therefore

$$
\begin{aligned}
\tilde{D}_{t, k} J_{k}^{t} & =\Gamma_{t} H_{t} R_{t} T_{k} \\
& =\tilde{O}_{t, k} .
\end{aligned}
$$

We then have that, for $t \geq L, O_{t, T, k} \in \mathcal{F C R} \Leftrightarrow \tilde{O}_{t, T, k} \in$ $\mathcal{F C R}$. Also, for $t<L$, we obtain

$$
\begin{aligned}
& \limsup _{T \rightarrow \infty} \mathbb{P}\left(O_{t, T, k} \notin \mathcal{F} \mathcal{C} \mathcal{R}\right)^{1 / T} \\
= & \limsup _{T \rightarrow \infty} \mathbb{P}\left(O_{t, L-t, k} \notin \mathcal{F C} \mathcal{C}\right)^{1 / T} \mathbb{P}\left(O_{L, T-L, k} \notin \mathcal{F C} \mathcal{R}\right)^{1 / T} \\
= & \limsup _{T \rightarrow \infty} \mathbb{P}\left(\tilde{O}_{L, T-L, k} \notin \mathcal{F} \mathcal{C} \mathcal{R}\right)^{1 / T} \\
= & \limsup _{T \rightarrow \infty} \mathbb{P}\left(\tilde{O}_{t, L-t, k} \notin \mathcal{F C} \mathcal{R}\right)^{1 / T} \mathbb{P}\left(\tilde{O}_{L, T-L, k} \notin \mathcal{F C} \mathcal{R}\right)^{1 / T} \\
= & \limsup _{T \rightarrow \infty} \mathbb{P}\left(\tilde{O}_{t, T, k} \notin \mathcal{F C} \mathcal{R}\right)^{1 / T},
\end{aligned}
$$

and the result follows. 


\section{B Proofs of Theorem 11}

Let

$$
E_{t, k}=\Gamma_{t}\left[\begin{array}{c}
H_{t}^{1} G_{k}^{1} \\
\vdots \\
H_{t}^{I} G_{k}^{I}
\end{array}\right]
$$

Clearly,

$$
\operatorname{rank}\left(\tilde{O}_{t, T, k}\right)=\operatorname{rank}\left(\left[\begin{array}{c}
E_{t, k} J_{k}^{t} \\
\vdots \\
E_{t+T-1, k} J_{k}^{t+T-1}
\end{array}\right]\right)
$$

It then follows from Lemma 8 that, for the purposes of computing $\Phi_{k}$, the pair $\left(J_{k}, \tilde{D}_{t, k}\right)$ is equivalent to $\left(J_{k}, E_{t, k}\right)$. For $S \in \mathbb{D}^{M}$ we use $\tilde{O}_{t, M, k}(S)$ to denote the value of $\tilde{O}_{t, M, k}$ resulting when $\Gamma_{t, M}=S$. Let $\mathcal{Z}_{t, k} \subset \mathbb{D}^{M}$ be the set of all $S \in \mathbb{D}^{M}$ such that $\tilde{O}_{t, M, k}(S) \notin \mathcal{F} \mathcal{C} \mathcal{R}$.

In order to compute $\Phi_{k}$, we make use of the result in $[15$, Proposition 24]. As with [15, Theorem 14], this result is stated under very general assumptions, which are guaranteed by the simpler assumptions given in [15, Proposition 18]. Again, by combining these two results we obtain the following lemma.

Lemma 21 (Combination of [15, Proposition 18] and [15, Proposition 24]) Consider a FMO block $\left(J_{k}, E_{t, k}\right)$. If $E_{t, k}$ is a statistically independent sequence of random matrices with discrete distribution and cyclostationary statistics, then

$$
\Phi_{k}=\max _{0 \leq t<P} \max _{S \in \mathcal{Z}_{t, k}} \varsigma_{t, k}(S)^{1 / M}
$$

where $M$ is defined in Theorem 6 and

$$
\varsigma_{t, k}(S)=\mathbb{P}\left\{\operatorname{ker}\left(\tilde{O}_{t, M, k}\left(\Gamma_{t, M}\right)\right) \supseteq \operatorname{ker}\left(\tilde{O}_{t, M, k}(S)\right)\right\}
$$

Clearly, the pair $\left(J_{k}, E_{t, k}\right)$ satisfies the conditions in Lemma 21. Hence we can use the result.

We say that node $i \in\{1, \cdots, I\}$ is complete with respect to $S \in \mathbb{D}^{M}$ and $k \in\{1, \cdots, K\}$ if $S$ includes $r_{k}=\left\lceil\operatorname{rank}\left(\Omega_{k}^{i}\right) / c_{i}\right\rceil$ measurements from node $i$. Let $\mathcal{C}(S)$ denote the set of complete nodes in $S$. We have that $\operatorname{ker}\left(\tilde{O}_{t, M, k}(\tilde{S})\right) \supseteq \operatorname{ker}\left(\tilde{O}_{t, M, k}(S)\right)$ if $\tilde{S}$ misses the same measurements on all nodes not in $\mathcal{C}(S)$. We then have

$$
\varsigma_{t, k}(S) \geq \prod_{i \notin \mathcal{C}(S)}\left(1-p_{i}\right)^{M-\nu^{i}(S)} .
$$

Let $\mathcal{N}(S)=\left\{0 \leq \nu^{i}(S) \leq M: i \notin \mathcal{C}(S)\right\}$. If $\mathcal{C}(S)$ is an insufficient set and $\mathcal{N}(S)$ are insufficient counts, then $S \in \mathcal{Z}_{t, k}$. Combining this with (B.1) and (B.2) we obtain

$$
\begin{aligned}
\Phi_{k} & \geq \max _{0 \leq t<P} \max _{\mathcal{L}} \max _{\text {insufficient } \mathcal{M}} \prod_{\text {insufficient }} \prod_{i \notin \mathcal{L}}\left(1-p_{i}\right)^{1-\frac{M^{i}}{M}} \\
& =\max _{\mathcal{L} \in \mathbb{L}_{k}} \max _{\mathcal{M} \in \mathbb{M}_{k}(\mathcal{L})} \prod_{i \notin \mathcal{L}}\left(1-p_{i}\right)^{1-\frac{M^{i}}{M}}
\end{aligned}
$$

Suppose that $L \geq d_{k}, k \in\{1, \cdots, K\}$ and $H_{t}, t \in \mathbb{N}$, are generated as in Assumption 10. Let $S \in \mathbb{D}^{M}$ be such that $\mathcal{C}(S) \in \mathbb{L}_{k}$ and $\mathcal{N}(S) \in \mathbb{M}_{k}(\mathcal{C}(S))$. Then, w.p.1 over the outcomes of $H_{t}$, any sequence $\tilde{S}$ obtained by adding to $S$ a new measurement from any node $i \notin \mathcal{C}(S)$ will yield $\tilde{O}_{t, M, k}(\tilde{S}) \in \mathcal{F C} \mathcal{R}$. It then follows that $\operatorname{ker}\left(\tilde{O}_{t, M, k}(\tilde{S})\right) \supseteq \operatorname{ker}\left(\tilde{O}_{t, M, k}(S)\right)$ if and only if $\tilde{S}$ misses the same measurements on all nodes which are incomplete with respect to $S$. We then have that (B.2) and (B.3) hold with equality, completing the proof.

\section{Proofs of Lemma 16}

We have

$$
\begin{aligned}
& P_{k P \mid k P-1} \\
\leq & \mathbb{E}\left[\left(x_{k P}-\hat{x}_{k P \mid(k-1) P-1}\right)\left(x_{k P}-\hat{x}_{k P \mid(k-1) P-1}\right)^{\top}\right] \\
= & A^{P} P_{(k-1) P \mid(k-1) P-1} A^{P \top}+\sum_{p=0}^{P-1} A^{p} Q A^{p \top} \\
\leq & \rho(A)^{2 P} P_{(k-1) P \mid(k-1) P-1}+\sum_{i=0}^{P-1} \rho(A)^{2 p} Q \\
\leq & \rho(A)^{2 P} P_{(k-1) P \mid(k-1) P-1}+\frac{\rho(A)^{2 P}}{\rho(A)^{2}-1} Q,
\end{aligned}
$$

and (16) follows since $P_{(k-1) P \mid(k-1) P-1} \geq Q$.

Fix $k$ and put $s=(k-1) P-L+1$. For any $(k-1) P \leq$ $t<k P$, it is straightforward to verify that

$$
\begin{aligned}
& x_{t}=A^{t-s} x_{s}+\epsilon_{t-1, s}, \\
& y_{t}=C A^{t-s} x_{s}+\varepsilon_{t, s},
\end{aligned}
$$

where

$$
\begin{aligned}
\epsilon_{t-1} & =\sum_{r=1}^{t-s} A^{r-1} w_{t-r} \\
\varepsilon_{t, s} & =C \epsilon_{t-1, s}+v_{t}
\end{aligned}
$$


Let $\bar{y}_{t}^{\top}=\left[y_{t-L+1}^{\top}, \cdots, y_{t}^{\top}\right]$ and $\bar{\varepsilon}_{t, s}^{\top}=$ $\left[\varepsilon_{t-L+1, s}^{\top}, \cdots, \varepsilon_{t, s}^{\top}\right]$. We then have

$$
\bar{y}_{t}=F A^{t-(k-1) P} x_{s}+\bar{\varepsilon}_{t, s} .
$$

Let $u_{t}=\Gamma_{t} z_{t}$. Then,

$$
\begin{aligned}
u_{t} & =\Gamma_{t} H_{t} \bar{y}_{t} \\
& =\Gamma_{t} H_{t} F A^{t-(k-1) P} x_{s}+\Gamma_{t} H_{t} \bar{\varepsilon}_{t, s} .
\end{aligned}
$$

Hence, letting $U_{k}^{\top}=\left[u_{(k-1) P}^{\top}, \cdots, u_{k P-1}^{\top}\right]$, we obtain

$$
U_{k}=\Xi_{k} x_{s}+m_{t, s}
$$

where

$$
m_{t, s}=\left[\begin{array}{c}
\Gamma_{(k-1) P} H_{(k-1) P} \bar{\varepsilon}_{(k-1) P, s} \\
\vdots \\
\Gamma_{k P-1} H_{k P-1} \bar{\varepsilon}_{k P-1, s}
\end{array}\right] .
$$

Using (C.1) and (C.2), we can obtain an estimate $\check{x}_{k P}$ of $x_{k P}$ as follows

$$
\check{x}_{k P}=A^{P} \Xi_{k}^{\dagger} U_{k}
$$

If $\Xi_{k}$ has full column rank, then

$$
\begin{aligned}
\check{x}_{k P} & =A^{P} \Xi_{k}^{\dagger}\left(\Xi_{k} x_{s}+m_{t, s}\right) \\
& =A^{P} x_{s}+\Xi_{k}^{\dagger} m_{t, s} .
\end{aligned}
$$

Then, using (C.1),

$$
\begin{aligned}
e_{k} & =x_{k P}-\check{x}_{k P} \\
& =\epsilon_{t-1, s}-m_{t, s} .
\end{aligned}
$$

Since $e_{k}$ is only formed by noise terms, it is straightforward to see that there exists a matrix $\bar{P}$ such that, w.p.1 and for all $k \in \mathbb{N}$,

$$
\mathbb{E}\left[e_{k} e_{k}^{\top}\right] \leq \bar{P}
$$

The result then follows after noticing that, since $\check{x}_{k P}$ is a sub-optimal estimator,

$$
P_{k P \mid k P-1} \leq \mathbb{E}\left[e_{k} e_{k}^{\top}\right]
$$

\section{Proofs of Theorem 18}

Let $q_{k}^{i}, k \in \mathbb{N}, i \in\{1, \cdots, I\}$ denote the number of measurements received from sensor $i$ during the time interval $[(k-1) P, k P]$. Let $\mathcal{Q}_{k}=\left\{q_{k}^{i}: i=1, \cdots, I\right\}$. For
$0 \leq l \leq k$, let $\mathcal{E}_{l, k}$ denote the event in which $\mathcal{Q}_{l} \in \mathbb{Q}$ and $\mathcal{Q}_{m} \notin \mathbb{Q}$, for all $m \in\{l+1, \cdots, k\}$. In particular, notice that $\mathcal{E}_{0, k}$ denotes the event in which $\mathcal{Q}_{m} \notin \mathbb{Q}$, for all intervals up to $[(k-1) P, k P]$.

Since $H_{t}, t \in \mathbb{N}$, is pseudo-randomly generated with period $P \geq n$, and $L \geq n$, it is straightforward to see that, with probability one over the outcomes of $H_{t}$, the matrix $Q_{l}$ has full column rank if $\mathcal{Q}_{l}$ is feasible. It then follows from Lemma 16 that

$$
\begin{aligned}
\mathbb{E}\left[P_{k P \mid k P-1}\right] & =\sum_{l=0}^{k} \mathbb{E}\left[P_{k P \mid k P-1} \mid \mathcal{E}_{l, k}\right] \mathbb{P}\left[\mathcal{E}_{l, k}\right] \\
& \leq \sum_{l=0}^{k} \kappa^{k-l} \mathbb{E}\left[P_{l P \mid l P-1} \mid \mathcal{E}_{l, k}\right] \mathbb{P}\left[\mathcal{E}_{l, k}\right] \\
& \leq \sum_{l=0}^{k} \kappa^{k-l} \mathbb{P}\left[\mathcal{E}_{l, k}\right] \bar{P}
\end{aligned}
$$

where

$$
\kappa=\rho(A)^{2 P} \frac{\rho(A)^{2}}{\rho(A)^{2}-1} .
$$

Let

We have

$$
\varpi_{P}=\mathbb{P}\left[\mathcal{Q}_{l} \notin \mathbb{Q}\right]
$$

$$
\mathbb{P}\left[\mathcal{E}_{l, k}\right]=\varpi_{P}^{k-l}\left(1-\varpi_{P}\right) .
$$

By listing all the possibilities of the event $\mathcal{Q}_{l} \notin \mathbb{Q}$, it follows that

$$
\mathbb{P}\left[\mathcal{Q}_{l} \notin \mathbb{Q}\right]=\sum_{\mathcal{Q} \notin \mathbb{Q}} \prod_{i=1}^{I}\left(\begin{array}{l}
P \\
q^{i}
\end{array}\right) p_{i}^{q_{i}}\left(1-p_{i}\right)^{P-q_{i}}
$$

Putting the above into (D.1) yields

$$
\mathbb{E}\left[P_{k P \mid k P-1}\right]<\left(1-\varpi_{P}\right) \bar{P} \sum_{l=0}^{k}\left(\kappa \varpi_{P}\right)^{k-l}
$$

Hence, $\sup _{k \in \mathbb{N}} \mathbb{E}\left[P_{k P \mid k P-1}\right]<\infty$ if

$$
\begin{aligned}
1 & \geq\left(\kappa \varpi_{P}\right)^{1 / P} \\
& =\rho(A)^{2}\left(\frac{\rho(A)^{2}}{\rho(A)^{2}-1}\right)^{1 / P} \varpi_{P}^{1 / P} \\
& =\rho(A)^{2} \pi_{P}^{1 / P},
\end{aligned}
$$

and (18) the result follows.

Suppose that $\left(A, C^{i}\right)$ is observable for each $i=1, \ldots, I$. Then, $\mathcal{Q} \notin \mathbb{Q}$ implies that $q^{i} \leq n$ for all $i=1,2, \ldots, I$. 
Thus, we have

$$
\lim _{P \rightarrow \infty} \pi_{P}^{1 / P}=\prod_{i=1}^{I}\left(1-p_{i}\right) .
$$

Recall from Section 4.1 that, for each $k=1, \ldots, K, \mathbb{L}_{k}$ denotes the collection of all maximal insufficient sets. Since all $\left(A, C^{i}\right)$ are observable, we have $\mathbb{L}_{k}=\emptyset$. Hence, from Corollary 14, we have

$$
\lim _{P \rightarrow \infty} \Phi_{k}=\prod_{i=1}^{I}\left(1-p_{i}\right)
$$

for all $k=1, \ldots, K$. Then, (19) follows from the necessary condition stated in Theorem 6 .

\section{References}

[1] V. Gupta, T. Chung, B. Hassibi, and R. Murray. On a stochastic sensor selection algorithm with applications in sensor scheduling and sensor coverage. Automatica, 42(2):251-260, 2006.

[2] X. Liu and A. Goldsmith. Kalman filtering with partial observation losses. In Proceedings of 43th IEEE Conference on Decision and Control, pages 4180-4186. IEEE, 2004.

[3] B. Sinopoli, L. Schenato, M. Franceschetti, K. Poolla, M. Jordan, and S. Sastry. Kalman filtering with intermittent observations. IEEE Transactions on Automatic Control, 49(9):1453-1464, 2004.

[4] L. Schenato, B. Sinopoli, M. Franceschetti, K. Poolla, and S. Sastry. Foundations of control and estimation over lossy networks. Proceedings of the IEEE, 95(1):163-187, 2007.

[5] J. Hespanha, P. Naghshtabrizi, and Y. Xu. A survey of recent results in networked control systems. Proceedings of the IEEE, 95(1):138-162, 2007.

[6] K. Plarre and F. Bullo. On Kalman filtering for detectable systems with intermittent observations. IEEE Transactions on Automatic Control, 54(2):386-390, 2009.

[7] M. Huang and S. Dey. Stability of Kalman filtering with Markovian packet losses. Automatica, 43(4):598-607, 2007.

[8] K. You, M. Fu, and L. Xie. Mean square stability for Kalman filtering with markovian packet losses. Automatica, 47(12):2647-2657, 2011.

[9] Y. Mo and B. Sinopoli. Towards finding the critical value for Kalman filtering with intermittent observations. http://arxiv.org/abs/1005.2442, 2010.

[10] Y. Mo and B. Sinopoli. A characterization of the critical value for kalman filtering with intermittent observations. In 47th IEEE Conference on Decision and Control, pages 26922697, 2008

[11] Y. Mo and B. Sinopoli. Kalman filtering with intermittent observations: Tail distribution and critical value. IEEE Transactions on Automatic Control, 57(3):677- 689, 2012.

[12] L. Xie and L. Xie. Peak covariance stability of a random Riccati equation arising from Kalman filtering with observation losses. Journal of Systems Science and Complexity, 20(2):262-272, 2007.
[13] E. Rohr, D. Marelli, and M. Fu. Kalman filtering for a class of degenerate systems with intermittent observations. In 50th IEEE Conference on Decision and Control and European Control Conference, Orlando, Florida, 2011.

[14] E. Rohr, D. Marelli, and M. Fu. Kalman filtering with intermittent observations: On the boundedness of the expected error covariance. IEEE Transactions on Automatic Control, 59(10):2724-2738, 2014.

[15] D. Marelli, T. Sui, E. Rohr, and M. Fu. Stability of Kalman Filtering with a random measurement equation: Application to sensor scheduling with intermittent observations. Automatica, 99:390-402, 2019.

[16] K. Okano and H. Ishii. Stabilization of uncertain systems using quantized and lossy observations and uncertain control inputs. Automatica, 81:261-269, 2017.

[17] F. Smarra, M. Benedetto, and A. Innocenzo. Efficient routing redundancy design over lossy networks. International Journal of Robust and Nonlinear Control, 28(6):2574-2597, 2018.

[18] L. Schenato. Optimal estimation in networked control systems subject to random delay and packet drop. IEEE Transactions on Automatic Control, 53(5):1311-1317, 2008.

[19] R. Koetter and M. Medard. An algebraic approach to network coding. IEEE/ACM Transactions on Networking, 11(5):782$795,2003$.

[20] E. Erez, M. Kim, Y. Xu, Yeh. E, and M. Medard. Deterministic network model revisited: An algebraic network coding approach. IEEE Transactions on Information Theory, 60(8):4867-4879, 2014.

[21] L. He, D. Han, X. Wang, and L. Shi. Optimal linear state estimation over a packet-dropping network using linear temporal coding. Automatica, 49(4):1075-1082, 2013.

[22] T. Sui, K. You, M. Fu, and D. Marelli. Stability of MMSE state estimators over lossy networks using linear coding. Automatica, 51(1):167-174, 2015.

[23] X. He, Z. Wang, X. Wang, and D. Zhou. Networked strong tracking filtering with multiple packet dropouts: algorithms and applications. IEEE Transactions on Industrial Electronics, 61(3):1454-1463, 2014.

[24] J. Hu, Z. Wang, and H. Gao. Recursive filtering with random parameter matrices, multiple fading measurements and correlated noises. Automatica, 49(11):3440-3448, 2013.

[25] J. Hu, Z. Wang, H. Gao, and L. Stergioulas. Extended kalman filtering with stochastic nonlinearities and multiple missing measurements. Automatica, 48(9):2007-2015, 2012.

[26] G. Wei, Z. Wang, and H. Shu. Robust filtering with stochastic nonlinearities and multiple missing measurements. Automatica, 45(3):836-841, 2009.

[27] S. Deshmukh, B. Natarajan, and A. Pahwa. State estimation over a lossy network in spatially distributed cyberphysical systems. IEEE Transactions on Signal Processing, 62(15):3911-3923, 2014.

[28] D. Quevedo, A. Ahlén, and K. Johansson. State estimation over sensor networks with correlated wireless fading channels. IEEE Transactions on Automatic Control, 58(3):581-593, 2013.

[29] K. Gatsis, M. Pajic, A. Ribeiro, and G. Pappas. Opportunistic control over shared wireless channels. IEEE Transactions on Automatic Control, 60(12):3140-3155, 2015.

[30] T. Sui, K. You, and M. Fu. Stability conditions for multisensor state estimation over a lossy network. Automatica, 53(3):1-9, 2015.

[31] B. Anderson and B. Moore. Optimal Filtering. Prentice-hall \& Systems Sciences Series, New Jersey, 1979. 